\title{
Majorizing measures and proportional subsets of bounded orthonormal systems
}

\section{Olivier Guédon, Shahar Mendelson, Alain Pajor and Nicole Tomczak-Jaegermann}

\begin{abstract}
In this article we prove that for any orthonormal system $\left(\varphi_{j}\right)_{j=1}^{n} \subset$ $L_{2}$ that is bounded in $L_{\infty}$, and any $1<k<n$, there exists a subset $I$ of cardinality greater than $n-k$ such that on $\operatorname{span}\left\{\varphi_{i}\right\}_{i \in I}$, the $L_{1}$ norm and the $L_{2}$ norm are equivalent up to a factor $\mu(\log \mu)^{5 / 2}$, where $\mu=\sqrt{n / k} \sqrt{\log k}$. The proof is based on a new estimate of the supremum of an empirical process on the unit ball of a Banach space with a good modulus of convexity, via the use of majorizing measures.
\end{abstract}

\section{Introduction}

We study some natural empirical processes determined by uniformly convex Banach spaces with modulus of convexity of power type 2. Results of this kind were extensively studied in a Hilbertian setting, and became an important tool for investigations, for example, of the behaviour of various random sets of vectors (as in $[14,15,7,16,2,6]$ ). We then apply these results to address a problem of selecting a subset of a bounded orthonormal system (for example, a set of characters), in the spirit of a result of Bourgain (see [20]) and of Talagrand [20], that also has applications to a sparse reconstruction $([2,16,6])$. A particular case of this result, formulated in Theorem 3 , is a so called Kashin's splitting of a set of $2 k$ orthonormal vectors in $L_{2}$ that is bounded in $L_{\infty}$.

2000 Mathematics Subject Classification: 46B07, 46B09, 42A05, $42 \mathrm{~A} 61$.

Keywords: Empirical process, majorizing measure, orthonormal system.g 
Theorem. There exist two positive constants $c$ and $C$ such that for any even integer $n$ and any orthonormal system $\left(\varphi_{j}\right)_{j=1}^{n}$ in $L_{2}$ with $\left\|\varphi_{j}\right\|_{L_{\infty}} \leq L$ for $1 \leq j \leq n$, we can find a subset $I \subset\{1, \ldots, n\}$ with $n / 2-c \sqrt{n} \leq|I| \leq$ $n / 2+c \sqrt{n}$ such that for every $a=\left(a_{i}\right) \in \mathbb{C}^{n}$,

$$
\left\|\sum_{i \in I} a_{i} \varphi_{i}\right\|_{L_{2}} \leq C L \sqrt{\log n}(\log \log n)^{5 / 2}\left\|\sum_{i \in I} a_{i} \varphi_{i}\right\|_{L_{1}}
$$

and

$$
\left\|\sum_{i \notin I} a_{i} \varphi_{i}\right\|_{L_{2}} \leq C L \sqrt{\log n}(\log \log n)^{5 / 2}\left\|\sum_{i \notin I} a_{i} \varphi_{i}\right\|_{L_{1}} .
$$

This result strengthens Theorem 2.4 of [6] and is almost optimal. We have been told by J. Bourgain that the term $\sqrt{\log n}$ is necessary. We would like to thank L. Rodriguez-Piazza for showing us the details of the proof of this optimality in the case of the Walsh system and for allowing us to present the argument at the end of this paper. We thank also the referee who remarks that this construction allows to show that this bound is almost optimal for the trigonometric system as well. The technical proof of Theorem 1 about empirical processes will be presented in the first part. It is based on a construction of majorizing measures developped in [14] and [7] and the main new ideas we use are some observations on packing and covering numbers.

\section{Maximal deviation of the empirical moment}

We begin this section with some definitions and notation. If $E$ is a normed space we denote by $E^{*}$ the dual space to $E$ and the dual norm is denoted by \|\|$_{*}$. The modulus of convexity of $E$ is defined for any $\varepsilon \in(0,2)$ by

$$
\delta_{E}(\varepsilon)=\inf \left\{1-\left\|\frac{x+y}{2}\right\|,\|x\|=1,\|y\|=1,\|x-y\|>\varepsilon\right\} .
$$

We say that $E$ has modulus of convexity of power type 2 if there is a constant $c$ such that $\delta_{E}(\varepsilon) \geq c \varepsilon^{2}$ for every $\varepsilon \in(0,2)$. It is well-known (see e.g., [11], Proposition 2.4) that this property is equivalent to the fact that the inequality

$$
\left\|\frac{x+y}{2}\right\|^{2}+\lambda^{-2}\left\|\frac{x-y}{2}\right\|^{2} \leq \frac{1}{2}\left(\|x\|^{2}+\|y\|^{2}\right)
$$

holds for all $x, y \in E$ (where $\lambda>0$ is a constant depending only on $c$ ). If (2.1) is satisfied then we say that $E$ has modulus of convexity of power type 2 with constant $\lambda$ (in such a case it is clear that $\delta_{E}(\varepsilon) \geq \varepsilon^{2} / 2 \lambda^{2}$ ). 
The notions of type (and cotype) of a Banach space were studied extensively during the 70's (see, for example, [10] and the survey [9]). A Banach space $E$ has type $p$ if there is a constant $C$ such that for every $N \in \mathbb{N}$ and every $x_{1}, \ldots, x_{N}$

$$
\mathbb{E}\left\|\sum_{i=1}^{N} g_{i} x_{i}\right\| \leq C\left(\sum_{i=1}^{N}\left\|x_{i}\right\|^{p}\right)^{1 / p}
$$

where $g_{1}, \ldots, g_{N}$ are standard independent gaussian variables (that is $g_{i} \sim$ $\mathcal{N}(0,1))$. The smallest constant $C$ for which $(2.2)$ holds is called the type $p$ constant of $E$ and is denoted by $T_{p}(E)$.

Moreover, it is well known that if $E$ has modulus of convexity of power type 2 then the dual space $E^{*}$ has also modulus of smoothness of power type 2 , and therefore, $E^{*}$ has type 2 (see, for example, [8, Theorem 1.e.16]).

\subsection{Results on empirical processes}

Our first theorem generalizes a result of Rudelson [15], proved in a Hilbertian setting, to the case of a Banach space with modulus of convexity of power type 2. In fact, in Theorem 1 we solve a question left open in [7] by removing the condition on the distance of $E$ to an Euclidean space of the same dimension.

Theorem 1. There exists an absolute constant $C$ for which the following holds. Let $E$ be a Banach space with modulus of convexity of power type 2 with constant $\lambda$. Then, for every vectors $X_{1}, \ldots, X_{m}$ in $E^{*}$,

$$
\begin{aligned}
& \left.\mathbb{E} \sup _{y \in B_{E}}\left|\sum_{j=1}^{m} \varepsilon_{j}\right|\left\langle X_{j}, y\right\rangle\right|^{2} \mid \leq \\
& \leq C \lambda^{4} T_{2}\left(E^{*}\right) \sqrt{\log m} \max _{1 \leq j \leq m}\left\|X_{j}\right\|_{*} \sup _{y \in B_{E}}\left(\sum_{j=1}^{m}\left|\left\langle X_{j}, y\right\rangle\right|^{2}\right)^{1 / 2},
\end{aligned}
$$

where the expectation is taken over the i.i.d. Bernoulli random variables $\left(\varepsilon_{j}\right)_{1 \leq j \leq m}$ and $T_{2}\left(E^{*}\right)$ is the type 2 constant of $E^{*}$.

The proof of Theorem 1 uses the same construction of a majorizing measure used in [14] and in [7], and this construction was inspired by the work of Talagrand in [18]. The improvement in Theorem 1 compared with [7] comes from entropy estimates that will be presented in Section 2.2. We will only sketch the construction of the majorizing measure in Section 2.3 and explain how the argument from [7] may be adapted using the new estimates. 
As it has been proved in [15], one can apply a symmetrization argument due to Giné and Zinn [5] combined with Theorem 1 and deduce the following result.

Theorem 2. There exists an absolute constant $C$ for which the following holds. Let $E$ be a Banach space with modulus of convexity of power type 2 with constant $\lambda$. Let $X$ be a random vector in $E$ and set $X_{1}, \ldots, X_{m}$ to be independent copies of $X$. If

$$
A=C \lambda^{4} T_{2}\left(E^{*}\right) \sqrt{\frac{\log m}{m}}\left(\mathbb{E} \max _{1 \leq j \leq m}\left\|X_{j}\right\|_{*}^{2}\right)^{1 / 2} \text { and } \sigma^{2}=\sup _{y \in B_{E}} \mathbb{E}|\langle X, y\rangle|^{2}
$$

then

$$
\left.\mathbb{E} \sup _{y \in B_{E}}\left|\frac{1}{m} \sum_{j=1}^{m}\right|\left\langle X_{j}, y\right\rangle\right|^{2}-\mathbb{E}|\langle X, y\rangle|^{2} \mid \leq A^{2}+\sigma A .
$$

We omit further details and refer the reader to one of the articles $[15,7$, $16,6]$.

\subsection{Covering and packing numbers}

The new ingredient of our proof, in comparison with [7], are estimates on packing and covering numbers which we shall now discuss.

Definition 1. Let $T$ and $B$ be symmetric convex bodies in a Banach space $E$. Define $N(T, B)$ to be the minimal number of translates of $B$ needed to cover $T$, i.e.

$$
N(T, B)=\inf \left\{N, \exists\left\{x_{1}, \ldots, x_{N}\right\} \subset T \text { such that } T \subset \cup_{j=1}^{N} x_{j}+B\right\} .
$$

We denote by $M(T, B)$ the maximal number of disjoint translates of $B$ by elements of $T$, i.e.

$$
M(T, B)=\sup \left\{N, \exists\left\{x_{1}, \ldots, x_{N}\right\} \subset T \text { such that } x_{j}-x_{k} \notin B \text { for } j \neq k\right\} .
$$

It is well known that these two quantities are related by

$$
N(T, B) \leq M(T, B) \leq N(T, B / 2) .
$$

Note that if $E$ is a normed space, $B_{E}$ is its unit ball and $B=\varepsilon B_{E}$, then $M\left(T, \varepsilon B_{E}\right)$ is the cardinality of a maximal $\varepsilon$-separated subset of $T$ with respect to the norm in $E$. Also, if $S: X \rightarrow Y$ is an operator, we sometimes denote $M\left(S B_{X}, \varepsilon B_{Y}\right)$ by $M(S: X \rightarrow Y, \varepsilon)$, and use a similar notation for the covering numbers $N$.

The following is a combination of results that appeared in [1] and in [3] (see Lemma 3.3 in [18]). We repeat its proof since we need precise estimates on the dependence on the modulus of convexity of the space $E$. 
Lemma 1. Let $E$ be a Banach space of modulus of convexity of power type 2 with constant $\lambda$ and denote by $T_{2}\left(E^{*}\right)$ the type 2 constant of $E^{*}$.

Let $X_{1}, \ldots, X_{m}$ be vectors in $E^{*}$ such that for every $1 \leq j \leq m,\left\|X_{j}\right\|_{*} \leq L$, and define for every $y \in E$,

$$
\|y\|_{\infty, m}=\max _{1 \leq \ell \leq m}\left|\left\langle X_{\ell}, y\right\rangle\right| .
$$

If $\varepsilon>0$ and $x_{1}, \ldots, x_{N} \in B_{E}$ are $\varepsilon$-separated with respect to $\|\cdot\|_{\infty, m}$ then

$$
\varepsilon \sqrt{\log N} \leq C \lambda^{2} T_{2}\left(E^{*}\right) L \sqrt{\log m}
$$

where $C$ is an absolute constant.

Proof. Let $\left(e_{i}\right)_{i=1}^{m}$ be the standard basis in $\ell_{1}^{m}$ and define $S: \ell_{1}^{m} \rightarrow E^{*}$ by $S e_{\ell}=X_{\ell}$ for every $\ell=1, \ldots, m$. Hence, for every $\ell \neq \ell^{\prime},\left|S^{*}\left(x_{\ell}-x_{\ell^{\prime}}\right)\right|_{\infty} \geq \varepsilon$ where $|\cdot|_{\infty}$ denotes the norm in $\ell_{\infty}^{m}$. Thus,

$$
N \leq M\left(S^{*}: E \rightarrow \ell_{\infty}^{m}, \varepsilon\right)
$$

Since $\|S\|=\max _{1 \leq \ell \leq N}\left\|X_{\ell}\right\|_{*} \leq L$ and $E^{*}$ has type 2, Proposition 1 in [3] yields that

$$
\varepsilon \sqrt{\log N\left(S: \ell_{1}^{m} \rightarrow E^{*}, \varepsilon\right)} \leq C L T_{2}\left(E^{*}\right) \sqrt{\log m}
$$

where $C$ is an absolute constant. By (2.4), a similar estimate holds for $M\left(S: \ell_{1}^{m} \rightarrow E^{*}, \varepsilon\right)$, where $C$ is replaced by a new constant, also denoted by $C$. Define the function

$$
f(\varepsilon)=\varepsilon \sqrt{\log M\left(S: \ell_{1}^{m} \rightarrow E^{*}, \varepsilon\right)}
$$

and observe that $f$ is bounded by $C L T_{2}\left(E^{*}\right) \sqrt{\log m}$.

Since $E$ has modulus of convexity of power type 2 with constant $\lambda$, Proposition 2 in [1] states that for every $\theta \geq \varepsilon / 5$,

$$
M\left(S^{*}: E \rightarrow \ell_{\infty}^{m}, \varepsilon\right) \leq M\left(S^{*}: E \rightarrow \ell_{\infty}^{m}, \theta\right) M\left(S: \ell_{1}^{m} \rightarrow E^{*}, c \varepsilon \delta_{E}(\varepsilon / 5 \theta)\right)
$$

and recall that $\delta_{E}(\varepsilon / \theta) \geq \varepsilon^{2} / 8 \lambda^{2} \theta^{2}$. Therefore, if

$$
h(\varepsilon)=\varepsilon \sqrt{\log M\left(S^{*}: E \rightarrow \ell_{\infty}^{m}, \varepsilon\right)},
$$

then the previous inequality implies that

$$
h(\varepsilon) \leq \frac{\varepsilon}{\theta} h(\theta)+c^{\prime}\left(\frac{\lambda^{2} \theta^{2}}{\varepsilon^{2}}\right) f\left(\frac{c \varepsilon^{3}}{\theta^{2} \lambda^{2}}\right)
$$

where $c, c^{\prime}$ are absolute constants. 
Choosing $\theta=2 \varepsilon$ and taking the supremum in $\varepsilon$, it follows that $h$ is bounded by

$$
C^{\prime} \lambda^{2} L T_{2}\left(E^{*}\right) \sqrt{\log m}
$$

proving the announced result.

The next lemma is a simple application of Sudakov's inequality [17].

Lemma 2. There exists an absolute constant $C$ for which the following holds. Let $E$ be a Banach space such that $E^{*}$ has type 2. Assume that $X_{1}, \ldots, X_{m}$ are vectors in $E^{*}$ with $\sup _{1 \leq j \leq m}\left\|X_{j}\right\|_{*} \leq$ L. Let $\alpha_{1}, \ldots, \alpha_{m}$ such that $\sum_{\ell=1}^{m} \alpha_{\ell}^{2} \leq 1$ and for every $y \in E$ set

$$
|y|_{\mathcal{E}}^{2}=\sum_{\ell=1}^{m}\left|\left\langle X_{\ell}, y\right\rangle\right|^{2} \alpha_{\ell}^{2}
$$

If $\varepsilon>0$ and $x_{1}, \ldots, x_{N} \in B_{E}$ are $\varepsilon$-separated with respect to $|\cdot|_{\mathcal{E}}$, then

$$
\varepsilon \sqrt{\log N} \leq C T_{2}\left(E^{*}\right) L .
$$

Proof . Let $\mathcal{E}_{1}$ be the ellipsoid in $\mathbb{R}^{m}$ consisting of all $y \in \mathbb{R}^{m}$ such that $|y|_{\mathcal{E}_{1}}:=\left(\sum_{\ell=1}^{m} \alpha_{\ell}^{2}\left\langle y, e_{\ell}\right\rangle^{2}\right)^{1 / 2} \leq 1$. Set $H=\left(\mathbb{R}^{m},|\cdot|_{\mathcal{E}_{1}}\right)$ and define the operator $S: H^{*} \rightarrow E^{*}$ by $S e_{i}=X_{i}$, where $\left(e_{i}\right)_{i=1}^{m}$ is the standard basis in $\mathbb{R}^{m}$. Note that for every $x_{i}, x_{j}$,

$$
\left|S^{*}\left(x_{i}-x_{j}\right)\right|_{\mathcal{E}_{1}}^{2}=\sum_{\ell=1}^{m} \alpha_{\ell}^{2}\left\langle S^{*}\left(x_{i}-x_{j}\right), e_{\ell}\right\rangle^{2}=\left|x_{i}-x_{j}\right|_{\mathcal{E}}^{2},
$$

and thus the points $\left\{S^{*}\left(x_{1}\right), \ldots, S^{*}\left(x_{N}\right)\right\}$ are $\varepsilon$-separated in $|\cdot|_{\mathcal{E}_{1}}$ and belong to $S^{*}\left(B_{E}\right)$. By $(2.4), N \leq N\left(S^{*}\left(B_{E}\right),(\varepsilon / 2) \mathcal{E}_{1}\right)$. On the other hand, $\mathcal{E}_{1}=T^{-1}\left(B_{2}^{m}\right)$ where $T: \mathbb{R}^{m} \rightarrow \mathbb{R}^{m}$ is the diagonal operator $T e_{\ell}=\alpha_{\ell} e_{\ell}$. Applying Sudakov's inequality [17],

$$
\varepsilon \sqrt{\log N} \leq \varepsilon \sqrt{\log N\left(S^{*}\left(B_{E}\right),(\varepsilon / 2) T^{-1}\left(B_{2}^{m}\right)\right)} \leq C \mathbb{E} \sup _{z \in T\left(S^{*}\left(B_{E}\right)\right)}\langle G, z\rangle
$$

where $G$ is a canonical Gaussian vector in $\ell_{2}^{m}$ and $C$ is an absolute constant. Moreover

$$
\begin{aligned}
& \mathbb{E} \sup _{z \in T\left(S^{*}\left(B_{E}\right)\right)}\langle G, z\rangle=\mathbb{E} \sup _{y \in B_{E}}\left\langle T G, S^{*} y\right\rangle \\
& =\mathbb{E}\left\|\sum_{\ell=1}^{m} \alpha_{\ell} g_{\ell} X_{\ell}\right\|_{*} \leq T_{2}\left(E^{*}\right)\left(\sum_{\ell=1}^{m} \alpha_{\ell}^{2}\left\|X_{\ell}\right\|_{*}^{2}\right)^{1 / 2} \leq T_{2}\left(E^{*}\right) L
\end{aligned}
$$

where we have used the type 2 inequality for $E^{*}$ and the fact that for every $1 \leq \ell \leq m,\left\|X_{\ell}\right\|_{*} \leq L$. The result now follows from (2.5). 


\subsection{Construction of a majorizing measure}

The construction that we present here is the same one that was presented in [14] and in [7]. Let $X_{1}, \ldots, X_{m}$ be $m$ fixed vectors in $E^{*}$ and define the random process $\left\{V_{y}: y \in B_{E}\right\}$ by

$$
V_{y}=\sum_{j=1}^{m} \varepsilon_{j}\left|\left\langle X_{j}, y\right\rangle\right|^{2}
$$

where $\varepsilon_{j}$ are independent symmetric Bernoulli random variables.

Our aim is to show that when $E$ has modulus of convexity of power type 2 ,

$$
\begin{aligned}
& \mathbb{E} \sup _{y \in B_{E}}\left|V_{y}\right| \leq \\
& \quad \leq C \lambda^{4} T_{2}\left(E^{*}\right) \max _{1 \leq j \leq m}\left\|X_{j}\right\|_{*} \sqrt{\log m}\left(\sup _{y \in B_{E}} \sum_{i=1}^{m}\left|\left\langle X_{j}, y\right\rangle\right|^{2}\right)^{1 / 2}
\end{aligned}
$$

for a suitable absolute constant $C$.

It is known that the process $\left\{V_{y}: y \in B_{E}\right\}$ satisfies a sub-Gaussian tail estimate, namely, that for every $y, \bar{y} \in E$ and any $t>0$,

$$
P\left(\left|V_{y}-V_{\bar{y}}\right| \geq t\right) \leq 2 \exp \left(-\frac{c t^{2}}{\tilde{d}^{2}(y, \bar{y})}\right)
$$

where

$$
\tilde{d}^{2}(y, \bar{y})=\sum_{j=1}^{m}\left(\left|\left\langle X_{j}, y\right\rangle\right|^{2}-\left|\left\langle X_{j}, \bar{y}\right\rangle\right|^{2}\right)^{2}
$$

and $c$ is an absolute constant.

It will be preferable to consider the following quasi-metric

$$
d^{2}(y, \bar{y})=\sum_{j=1}^{m}\left|\left\langle X_{j}, y-\bar{y}\right\rangle\right|^{2}\left(\left|\left\langle X_{j}, y\right\rangle\right|^{2}+\left|\left\langle X_{j}, \bar{y}\right\rangle\right|^{2}\right)
$$

and the quasi-norm $\|\cdot\|_{\infty, m}$ endowed on $E$ by

$$
\|x\|_{\infty, m}=\max _{1 \leq j \leq m}\left|\left\langle X_{j}, x\right\rangle\right|
$$

The proof of inequality (2.6) is based on the majorizing measure theory of Talagrand [19]. The following theorem is a combination of Proposition 2.3, Theorem 4.1 and Proposition 4.4 of [19]. 
Theorem [19] Assume that the process $\left\{V_{y}: y \in B_{E}\right\}$ is subgaussian with respect to a metric $d$. Let $r \geq 2$ and $k_{0} \in \mathbb{Z}$ be the largest integer such that $r^{-k_{0}}$ is greater than the radius of $B_{E}$ with respect to the metric d. For every $k \geq k_{0}$ let $\phi_{k}: B_{E} \rightarrow \mathbb{R}^{+}$be a family of maps satisfying the following assumption: there exists $A>0$ such that for any point $x \in B_{E}$, any $k \geq k_{0}$ and any $N \in \mathbb{N}$

$(H)\left\{\begin{array}{l}\text { for any points } x_{1}, \ldots, x_{N} \in \mathcal{B}_{r^{-k}}(x) \text { with } d\left(x_{i}, x_{j}\right) \geq r^{-k-1}, i \neq j \\ \text { we have } \max _{i=1, \ldots, N} \phi_{k+2}\left(x_{i}\right) \geq \phi_{k}(x)+\frac{1}{A} r^{-k} \sqrt{\log N}\end{array}\right.$

Then

$$
\mathbb{E} \sup _{y \in B_{E}}\left|V_{y}-V_{0}\right| \leq c A \cdot \sup _{k \geq k_{0}, x \in K} \phi_{k}(x) .
$$

The construction requires certain properties of the quasi metric $d$ and the quasi norm \|\|$_{\infty, m}$. We refer to Propositions 1 and 2 in [7] for precise properties of these metrics and list the ones we require in the following lemma.

Lemma 3. For every $y, \bar{y} \in \mathbb{R}^{n}$ and every $u \in B_{E}$,

$$
\begin{aligned}
& \tilde{d}(y, \bar{y}) \leq 2 d(y, \bar{y}), \\
& d(y, \bar{y}) \leq \sqrt{2}\|y-\bar{y}\|_{\infty, m} \sqrt{M}, \\
& \|y-\bar{y}\|_{\infty, m} \leq \max _{1 \leq j \leq m}\left\|X_{j}\right\|_{*}\|y-\bar{y}\|, \\
& d^{2}(z, \bar{z}) \leq 8\left(|z-\bar{z}|_{\mathcal{E}_{u}}^{2}+M\|z-\bar{z}\|_{\infty, m}^{2}\left(\|z-u\|^{2}+\|\bar{z}-u\|^{2}\right)\right),
\end{aligned}
$$

where

$$
|x|_{\mathcal{E}_{u}}^{2}=\sum_{i=1}^{m}\left\langle X_{i}, x\right\rangle^{2}\left\langle X_{i}, u\right\rangle^{2} \text { and } M=\sup _{y \in B_{E}} \sum_{j=1}^{m}\left|\left\langle X_{j}, y\right\rangle\right|^{2} .
$$

Moreover, for every $x \in E$ and $\rho>0$, the ball (with respect to the quasimetric d) centered in $x$ and with radius $\rho$, denoted by $\mathcal{B}_{x}(\rho)$, is convex.

Note that by combining (2.8) and (2.9) it follows that for every $\rho>0$ and every $x \in B_{E}, \inf _{y \in \mathcal{B}_{\rho}(x)}\|y\|$ is attained.

Proof of Theorem 1. Since there is only a finite number of points $X_{1}, \ldots, X_{m}$ then by passing to a quotient of $E$ we can assume that $E$ is a finite dimensional space. We will denote its dimension by $n$ and obviously one can assume that $m \geq n$. Also, recall that if $E$ is a Banach space with modulus of convexity of power type 2 with constant $\lambda$ then every quotient of $E$ satisfies that property with a constant smaller than $\lambda$ (see, e.g., [8]). 
By the homogeneity of the statement we can assume that

$$
\sup _{y \in B_{E}} \sum_{j=1}^{m}\left|\left\langle X_{j}, y\right\rangle\right|^{2}=1
$$

and by inequality (2.7), $V_{y}$ is a sub-Gaussian process with the quasi-metric $2 d$.

Therefore, if we denote

$$
L=\max _{1 \leq j \leq m}\left\|X_{j}\right\|_{*}
$$

our aim is to show that

$$
\mathbb{E} \sup _{y \in B_{E}}\left|V_{y}\right| \leq C \lambda^{4} T_{2}\left(E^{*}\right) L \sqrt{\log m}
$$

for an absolute constant $C$.

By inequality (2.8), the diameter of $B_{E}$ with respect to the metric $d$ is bounded by $2 \sqrt{2} L$. Let $r$ be a fixed number chosen large enough, set $k_{0}$ to be the largest integer such that $r^{-k_{0}} \geq 2 \sqrt{2} L$ and put $k_{1}$ to be the smallest integer such that $r^{-k_{1}} \leq L / \sqrt{n}$, where $n$ is the dimension of $E$. We shall use the same definition of the functionals $\phi_{k}: B_{E} \rightarrow \mathbb{R}^{+}$as in [14] and [7], namely:

$$
\left\{\begin{array}{l}
\forall k \geq k_{1}+1, \phi_{k}(x)=1+\frac{1}{2 \log r}+\frac{\sqrt{n}}{L \sqrt{\log m}} \sum_{\ell=k_{1}}^{k} r^{-\ell} \sqrt{\log \left(1+4 L r^{\ell}\right)} \\
\forall k_{0} \leq k \leq k_{1}, \phi_{k}(x)=\min \left\{\|y\|^{2}, y \in \mathcal{B}_{8 r^{-k}}(x)\right\}+\frac{k-k_{0}}{\log m} .
\end{array}\right.
$$

It is easy to verify using definitions of $k_{0}$ and $k_{1}$ that

$$
\sup _{x \in B_{E}, k \geq k_{0}} \phi_{k}(x) \leq c
$$

where $c$ is an absolute constant.

It remains to prove that our functionals satisfy condition $(H)$ for

$$
A=C \lambda^{4} T_{2}\left(E^{*}\right) L \sqrt{\log m},
$$

and that will conclude the proof of Theorem 1.

Proof of condition $(H)$. Fix integers $N$ and $k$, let $x \in B_{E}$ and $x_{1}, \ldots, x_{N} \in$ $\mathcal{B}_{r^{-k}}(x)$ for which $d\left(x_{i}, x_{j}\right) \geq r^{-k-1}$.

For $k \geq k_{1}-1$, we always have

$$
\phi_{k+2}\left(x_{i}\right)-\phi_{k}(x) \geq \frac{\sqrt{n \log \left(1+4 L r^{k+2}\right)}}{L \sqrt{\log m}} r^{-k-2} .
$$


Since the points $x_{1}, \ldots, x_{N}$ are well separated with respect to the metric $d$, then by (2.8) and (2.9),

$$
\left\|x_{i}-x_{j}\right\| \geq r^{-k-1} / L \sqrt{2}
$$

By a classical volumetric estimate (see, for example [12]), $N\left(B_{E}, t B_{E}\right) \leq$ $(1+2 / t)^{n}$ where $n$ is the dimension of $E$. Therefore,

$$
\sqrt{\log N} \leq \sqrt{n \log \left(1+2 \sqrt{2} L r^{k+1}\right)}
$$

which proves the desired inequality.

The case $k_{0} \leq k \leq k_{1}-2$ is more delicate and the main ingredients in this part are the entropy estimates proved in part 2.2.

For $j=1, \ldots, N$ denote by $z_{j} \in B_{E}$ points at which $\min \left\{\|y\|^{2}, y \in\right.$ $\left.\mathcal{B}_{8 r^{-k-2}}\left(x_{j}\right)\right\}$ is attained and set $u \in B_{E}$ to be a point at which $\min \left\{\|y\|^{2}, y \in\right.$ $\left.\mathcal{B}_{8 r^{-k}}(x)\right\}$ is attained. Put

$$
\theta=\max _{j}\left\|z_{j}\right\|^{2}-\|u\|^{2}
$$

and then $\max _{j} \phi_{k+2}\left(x_{j}\right)-\phi_{k}(x)=\theta+\frac{2}{\log m}$. We shall prove that

$$
\theta+\frac{2}{\log m} \geq r^{-k} \sqrt{\log N} / A
$$

Following [7], it is evident from the properties of $\mathcal{B}_{\rho}$ (see Proposition 1 and 2 in [7] and Lemma 3) that for any $i \neq j, d\left(z_{i}, z_{j}\right) \geq c r^{-k-1}$ and that $d\left(x, z_{j}\right) \leq 8 r^{-k}$. It implies that $\left(z_{j}+u\right) / 2 \in \mathcal{B}_{8 r^{-k}}(x)$ and by the definition of $u,\|u\| \leq\left\|z_{j}+u\right\| / 2$. Since $B_{E}$ has modulus of convexity of power type 2 , then for all $j=1, \ldots, N\left\|z_{j}-u\right\| \leq \lambda \sqrt{2 \theta}$. Thus, the set

$$
U=u+\lambda \sqrt{2 \theta} B_{E}
$$

contains all the $z_{j}$ 's.

Fix an absolute constant $\tilde{c}$ to be named later, set

$$
\delta=\tilde{c} \lambda^{-1} r^{-k} \theta^{-1 / 2}
$$

and let $S$ be the maximal number of points in $U$ that are $\delta$ separated in $\|\cdot\|_{\infty, m}$. By Lemma 1 ,

$$
\delta \sqrt{\log S} \leq C \lambda^{2} T_{2}\left(E^{*}\right) L \sqrt{\log m} \lambda \sqrt{\theta}
$$

where $C$ is an absolute constant. We consider now two cases. 
First, assume that $S \geq \sqrt{N}$. Then by the previous estimate and the definition of $\delta$,

$$
\sqrt{\log N} \leq C T_{2}\left(E^{*}\right) L \sqrt{\log m} \lambda^{3} \sqrt{\theta} / \delta \leq C \theta \quad r^{k} \lambda^{4} T_{2}\left(E^{*}\right) L \sqrt{\log m}
$$

which proves (2.12).

The second case is when $S \leq \sqrt{N}$. Since $S$ is the maximal number of points in $U$ that are $\delta$ separated with respect to $\|\cdot\|_{\infty, m}, U$ is covered by $S$ balls of diameter smaller than $\delta$ in $\|\cdot\|_{\infty, m}$. Thus, there exists a subset $J$ of $\{1, \ldots, N\}$ with cardinality $|J| \geq \sqrt{N}$ such that

$$
\forall i, j \in J, \quad\left\|z_{i}-z_{j}\right\|_{\infty, m} \leq \delta
$$

Recall that for any $y \in E$,

$$
|y|_{\mathcal{E}_{u}}^{2}=\sum_{\ell=1}^{m}\left|\left\langle X_{\ell}, y\right\rangle\right|^{2}\left|\left\langle X_{\ell}, u\right\rangle\right|^{2}=\sum_{\ell=1}^{m}\left|\left\langle X_{\ell}, y\right\rangle\right|^{2} \alpha_{\ell}^{2} .
$$

It is evident from (2.11) that $\sum \alpha_{\ell}^{2} \leq 1$ and from (2.10) that for every $z, \bar{z}, u \in E$,

$$
d^{2}(z, \bar{z}) \leq 8\left(|z-\bar{z}|_{\mathcal{E}_{u}}^{2}+\|z-\bar{z}\|_{\infty, m}^{2}\left(\|z-u\|^{2}+\|\bar{z}-u\|^{2}\right)\right)
$$

Since $d\left(z_{i}, z_{j}\right) \geq c r^{-k-1}$,

$$
\left\|z_{i}-u\right\| \leq 2 \lambda \sqrt{\theta} \quad \text { and } \quad\left\|z_{i}-z_{j}\right\|_{\infty, m} \leq \delta
$$

for any $i, j \in J$, we can define $\tilde{c}$ small enough such that for all $i \neq j \in J$,

$$
\left|z_{i}-z_{j}\right|_{\mathcal{E}_{u}} \geq c r^{-k-1}
$$

Thus, there are $|J|$ points in $u+\lambda \sqrt{2 \theta} B_{E}$ that are $c r^{k-1}$-separated for $|\cdot|_{\mathcal{E}_{u}}$. Using Lemma 2,

$$
\sqrt{\log |J|} \leq C T_{2}\left(E^{*}\right) L r^{k} \lambda \sqrt{\theta}
$$

and a simple application of the arithmetic-geometric means inequality proves that

$$
\sqrt{\log N} \leq C T_{2}\left(E^{*}\right) L r^{k} \lambda \sqrt{\log m}\left(\theta+\frac{2}{\log m}\right)
$$

completing the proof of $(2.12)$ (because $\lambda \geq 1)$. 


\section{Selecting an arbitrary proportion of a bounded or- thonormal system}

In this section, we will prove the

Theorem 3. There exist two positive constants $c, C$ such that for any orthonormal system $\left(\varphi_{j}\right)_{j=1}^{n}$ in $L_{2}$ with $\left\|\varphi_{j}\right\|_{L_{\infty}} \leq L$ for $1 \leq j \leq n$, the following holds.

1) For any $1<k<n$ there exists a subset $I \subset\{1, \ldots, n\}$ with $|I| \geq n-k$ such that for every $a=\left(a_{i}\right) \in \mathbb{C}^{n}$,

$$
\left\|\sum_{i \in I} a_{i} \varphi_{i}\right\|_{L_{2}} \leq C \mu(\log \mu)^{5 / 2}\left\|\sum_{i \in I} a_{i} \varphi_{i}\right\|_{L_{1}}
$$

where $\mu=L \sqrt{n / k} \sqrt{\log k}$.

2) Moreover, if $n$ is an even integer, there exists a subset $I \subset\{1, \ldots, n\}$ with $n / 2-c \sqrt{n} \leq|I| \leq n / 2+c \sqrt{n}$ such that for every $a=\left(a_{i}\right) \in \mathbb{C}^{n}$,

$$
\left\|\sum_{i \in I} a_{i} \varphi_{i}\right\|_{L_{2}} \leq C L \sqrt{\log n}(\log \log n)^{5 / 2}\left\|\sum_{i \in I} a_{i} \varphi_{i}\right\|_{L_{1}}
$$

and

$$
\left\|\sum_{i \notin I} a_{i} \varphi_{i}\right\|_{L_{2}} \leq C L \sqrt{\log n}(\log \log n)^{5 / 2}\left\|\sum_{i \notin I} a_{i} \varphi_{i}\right\|_{L_{1}} .
$$

Remark. Theorem 3 is satisfactory when $k$ is an arbitrary proportion of $n$. It strengthens Theorem A from [6] and extends the result of Talagrand [20] which is applicable only when $|I|=n-k$ is a sufficiently small proportion of $n$. Here we get an arbitrary proportion, with the distance $\sqrt{\log n}(\log \log n)^{5 / 2}$, which is the right power of $\log n$ as we will see at the end of the paper.

Define $\rho=\rho_{k, n}$, the restricted Kolmogorov $k$-width of the system as the smallest number $\rho$ such that there exists a subset $I \subset\{1, \ldots, n\}$ with $|I| \geq n-k$ satisfying

$$
\left\|\sum_{i \in I} a_{i} \varphi_{i}\right\|_{L_{2}} \leq \rho\left\|\sum_{i \in I} a_{i} \varphi_{i}\right\|_{L_{1}}
$$

for every $a=\left(a_{i}\right) \in \mathbb{C}^{n}$ (see [6] section 3). It was proved in [2] that $\rho=$ $O\left(\sqrt{n / k} \log ^{3} n\right)$. This result was improved to $\rho=O\left(\sqrt{n / k} \sqrt{\log n} \log ^{3 / 2} k\right)$ in $[16]$. 
The proof of Theorem 3 is based on a random method and follows the argument given in [6] for proving Theorem 2.1. However, instead of working with the space $L_{1}$, which is not uniformly convex, we will approximate it by an $L_{p}$ space for $p$ "close" to 1 and use the full strength of the estimate given in Theorem 2.

Proposition 1. There exist two positive constants $c, C$ such that for any orthonormal system $\left(\varphi_{j}\right)_{j=1}^{n}$ in $L_{2}$ with $\left\|\varphi_{j}\right\|_{L_{\infty}} \leq L$ for $1 \leq j \leq n$, the following holds.

1) For any $p \in(1,2)$ and any $1<k<n$ there exists a subset $I \subset$ $\{1, \ldots, n\}$ with $|I| \geq n-k$ such that for every $a=\left(a_{i}\right) \in \mathbb{C}^{n}$,

$$
\left\|\sum_{i \in I} a_{i} \varphi_{i}\right\|_{L_{2}} \leq \frac{C}{(p-1)^{5 / 2}} L \sqrt{n / k} \sqrt{\log k}\left\|\sum_{i \in I} a_{i} \varphi_{i}\right\|_{L_{p}} .
$$

2) Moreover, if $n$ is an even integer, there exists a subset $I \subset\{1, \ldots, n\}$ with $n / 2-c \sqrt{n} \leq|I| \leq n / 2+c \sqrt{n}$ such that for every $a=\left(a_{i}\right) \in \mathbb{C}^{n}$,

$$
\left\|\sum_{i \in I} a_{i} \varphi_{i}\right\|_{L_{2}} \leq \frac{C}{(p-1)^{5 / 2}} L \sqrt{n / k} \sqrt{\log k}\left\|\sum_{i \in I} a_{i} \varphi_{i}\right\|_{L_{p}}
$$

and

$$
\left\|\sum_{i \notin I} a_{i} \varphi_{i}\right\|_{L_{2}} \leq \frac{C}{(p-1)^{5 / 2}} L \sqrt{n / k} \sqrt{\log k}\left\|\sum_{i \notin I} a_{i} \varphi_{i}\right\|_{L_{p}} .
$$

Proof of Proposition 1. Let $X$ be the random vector taking the value $\varphi_{i}$ with probability $1 / n$ and denote by $E$ the complex vectorial space spanned by $\left\{\varphi_{1}, \ldots, \varphi_{n}\right\}$. Let $1<k<n$ and let $X_{1}, \ldots, X_{k}$ be independent copies of $X$. We define an operator $\Gamma: E \rightarrow \ell_{2}^{k}$ by

$$
\forall y \in E, \quad \Gamma y=\sum_{i=1}^{k}\left\langle X_{i}, y\right\rangle e_{i}
$$

where $\left(e_{1}, \ldots, e_{k}\right)$ denotes the canonical basis of $\ell_{2}^{k}$. Since $\left(\varphi_{j}\right)_{j=1}^{n}$ is an orthonormal system of $L_{2}$, the basic properties of $\Gamma$ are:

$$
\left\{\begin{array}{l}
(i) \mathbb{E}\|\Gamma y\|_{\ell_{2}^{k}}^{2}=\frac{k}{n}\|y\|_{L_{2}}^{2}, \\
(i i) \operatorname{ker} \Gamma=\operatorname{span}\left\{\left(\varphi_{j}\right)_{j=1}^{n} \backslash\left(X_{i}\right)_{i=1}^{k}\right\}
\end{array}\right.
$$

We shall first prove that for any $\delta \in(0,1)$, with probability greater than $1-\delta$, the set $\left(\varphi_{i}\right)_{i \in I}=\left\{\left(\varphi_{j}\right)_{j=1}^{n} \backslash\left(X_{i}\right)_{i=1}^{k}\right\}$ satisfies

$$
\forall\left(a_{i}\right)_{i \in I} \in \mathbb{C}^{|I|},\left\|\sum_{i \in I} a_{i} \varphi_{i}\right\|_{L_{2}} \leq \frac{C}{\delta(p-1)^{5 / 2}} L \sqrt{n / k} \sqrt{\log k}\left\|\sum_{i \in I} a_{i} \varphi_{i}\right\|_{L_{p}}
$$

for a universal constant $C$. 
Let $S_{L_{2}}=\left\{y:\|y\|_{L_{2}}=1\right\}$ be the unit sphere in $L_{2}$ and observe that for any star-shaped subset $T \subset L_{2}$ the following holds: if $\rho>0$ satisfies

$$
\sup _{y \in T \cap \rho S_{L_{2}}}\left|\sum_{j=1}^{k}\left\langle X_{j}, y\right\rangle^{2}-\frac{k}{n} \rho^{2}\right| \leq \frac{k \rho^{2}}{3 n}
$$

then

$$
\operatorname{diam}(\operatorname{ker} \Gamma \cap T) \leq \rho .
$$

Indeed, condition (3.4) implies that for all $y \in T \cap \rho S_{L_{2}}$,

$$
\frac{2 k \rho^{2}}{3 n} \leq\|\Gamma y\|_{\ell_{2}^{k}}^{2} \leq \frac{4 k \rho^{2}}{3 n} .
$$

The homogeneity of (3.6) and the fact that $T$ is star-shaped imply that if the lower bound in (3.6) holds for all $y \in T \cap \rho S_{L_{2}}$, then the same lower bound also holds for all $y \in T$ with $\|y\|_{L_{2}} \geq \rho$. This in turn shows that if $y \in \operatorname{ker} \Gamma \cap T$ then $\|y\|_{L_{2}} \leq \rho$, as required in (3.5).

Since $\operatorname{ker} \Gamma=\operatorname{span}\left\{\left(\varphi_{j}\right)_{j=1}^{n} \backslash\left(X_{i}\right)_{i=1}^{k}\right\}$, we get for $T=B_{L_{p}} \cap E$ that whenever $\rho$ satisfies (3.4), then

$$
\left\|\sum_{i \in I} a_{i} \varphi_{i}\right\|_{L_{2}} \leq \rho\left\|\sum_{i \in I} a_{i} \varphi_{i}\right\|_{L_{p}}
$$

for all scalars $a_{i}$, proving that (3.3) is satisfied with the constant $\rho$.

In order to find $\rho$ that satisfies (3.4) with positive probability we use Theorem 2. Denote by $E_{p}$ the complex Banach space spanned by $\varphi_{1}, \ldots, \varphi_{n}$ endowed with the norm defined by

$$
\|y\|=\left(\frac{\|y\|_{L_{p}}^{2}+\rho^{-2}\|y\|_{L_{2}}^{2}}{2}\right)^{1 / 2} .
$$

It is clear that $\left(B_{L_{p}} \cap E\right) \cap \rho B_{L_{2}} \subset B_{E_{p}} \subset \sqrt{2}\left(B_{L_{p}} \cap E\right) \cap \rho B_{L_{2}}$ and that the following properties are satisfied:

$$
\left\{\begin{array}{l}
E_{p} \text { is a Banach space with modulus of convexity of power type } 2 \\
\text { with constant } \lambda^{-2}=p(p-1) / 8 \\
E_{p}^{*} \text { is a Banach space of type } 2 \text { and } T_{2}\left(E_{p}^{*}\right) \leq C \sqrt{q}=C \sqrt{p /(p-1)} .
\end{array}\right.
$$

Indeed, the first property follows from Clarkson inequality [4], that for any $f, g \in L_{p}$,

$$
\left\|\frac{f+g}{2}\right\|_{L_{p}}^{2}+\frac{p(p-1)}{8}\left\|\frac{f-g}{2}\right\|_{L_{p}}^{2} \leq \frac{1}{2}\left(\|f\|_{L_{p}}^{2}+\|g\|_{L_{p}}^{2}\right) .
$$

The second property is evident because for any $q \geq 2, L_{q}$ has type 2 with constant $C \sqrt{q}$. 
By property $(i)$ of $\Gamma$, we get, taking $T=B_{L_{p}} \cap E$ and applying Theorem 2 to $E_{p}$,

$$
\begin{aligned}
\mathbb{E} \sup _{y \in T \cap \rho S_{L_{2}}}\left|\sum_{j=1}^{k}\left\langle X_{j}, y\right\rangle^{2}-\frac{k}{n} \rho^{2}\right| & =\mathbb{E} \sup _{y \in T \cap \rho S_{L_{2}}}\left|\sum_{j=1}^{k}\left(\left\langle X_{j}, y\right\rangle^{2}-\mathbb{E}\left\langle X_{j}, y\right\rangle^{2}\right)\right| \\
& \leq \mathbb{E} \sup _{y \in T \cap \rho B_{L_{2}}}\left|\sum_{j=1}^{k}\left(\left\langle X_{j}, y\right\rangle^{2}-\mathbb{E}\left\langle X_{j}, y\right\rangle^{2}\right)\right| \\
& \leq \mathbb{E} \sup _{y \in B_{E_{p}}}\left|\sum_{j=1}^{k}\left(\left\langle X_{j}, y\right\rangle^{2}-\mathbb{E}\left\langle X_{j}, y\right\rangle^{2}\right)\right| \\
& \leq k\left(A^{2}+A \sigma\right)
\end{aligned}
$$

where $\sigma=\sup _{y \in B_{E_{p}}}\|y\|_{L_{2}} / \sqrt{n} \leq \sqrt{2} \rho / \sqrt{n}$ and

$$
\begin{aligned}
A & =C \lambda^{4} T_{2}\left(E_{p}^{*}\right) \sqrt{\frac{\log k}{k}}\left(\mathbb{E} \max _{j \in J}\left\|\varphi_{j}\right\|_{E_{p}^{*}}^{2}\right)^{1 / 2} \\
& \leq C(p-1)^{-5 / 2} L \sqrt{\frac{\log k}{k}}
\end{aligned}
$$

since for every $1 \leq j \leq n,\left\|\varphi_{j}\right\|_{E_{p}^{*}} \leq\left\|\varphi_{j}\right\|_{L_{\infty}} \leq L$. By Chebychev inequality, for any $\delta \in(0,1)$, we conclude that with probability greater than $1-\delta$, there exists $X_{1}, \ldots, X_{k}$ such that for any positive $\rho$,

$$
\sup _{y \in T \cap \rho S_{L_{2}}}\left|\|\Gamma y\|_{\ell_{2}^{k}}^{2}-\frac{k}{n} \rho^{2}\right| \leq \frac{k}{\delta}\left(A^{2}+A \sigma\right) .
$$

To conclude, we choose a constant $c$ large enough such that, for

$$
\rho=c \sqrt{\frac{n}{k}} \sqrt{\log k} \frac{L}{\delta(p-1)^{5 / 2}},
$$

the inequality (3.4) is satisfied with probability greater than $1-\delta$.

The cardinality of the set $\left(\varphi_{i}\right)_{i \in I}=\left\{\left(\varphi_{j}\right)_{j=1}^{n} \backslash\left(X_{i}\right)_{i=1}^{k}\right\}$ is greater than $n-k$ and the first part of the proposition is proven choosing $\delta=1 / 2$.

To prove the second part of the proposition, we make two more observations. First, if $\rho>0$ satisfies (3.4) then we have proved that (3.6) holds true. The upper estimate of this inequality implies that for all $y \in T \cap \rho S_{L_{2}}$,

$$
\sum_{i \in I}\left\langle\varphi_{i}, y\right\rangle^{2} \geq \sum_{i=1}^{n}\left\langle\varphi_{i}, y\right\rangle^{2}-\sum_{j=1}^{k}\left\langle X_{j}, y\right\rangle^{2} \geq \rho^{2}\left(1-\frac{4 k}{3 n}\right) .
$$


Since $T$ is star shaped, we conclude as before that this inequality holds for all $y \in T$ for which $\|y\|_{L_{2}} \geq \rho$. If $k<3 n / 4$ then we have proved that if $y \in T$ and $\left\langle\varphi_{i}, y\right\rangle=0$ for all $i \in I$ (i.e. $\left.y \in T \cap(\operatorname{ker} \Gamma)^{\perp}\right)$, then $\|y\|_{L_{2}} \leq \rho$. But $(\operatorname{ker} \Gamma)^{\perp}=\operatorname{span}\left\{\left(\varphi_{i}\right)_{i \notin I}\right\}$ and we conclude that if $\rho>0$ satisfies (3.4) with $k<3 n / 4$ and $T=B_{L_{p}} \cap E$ then for any $\left(a_{i}\right)_{i=1}^{n} \in \mathbb{C}^{n}$,

$$
\left\|\sum_{i \in I} a_{i} \varphi_{i}\right\|_{L_{2}} \leq \rho\left\|\sum_{i \in I} a_{i} \varphi_{i}\right\|_{L_{p}}
$$

and

$$
\left\|\sum_{i \notin I} a_{i} \varphi_{i}\right\|_{L_{2}} \leq \rho\left\|\sum_{i \notin I} a_{i} \varphi_{i}\right\|_{L_{p}} .
$$

Secondly, it is not difficult to prove with a combinatorial argument (see Lemma 1.3 in [6]) that if $k=[\lambda n]$ with $\lambda=\log 2<3 / 4$ then with probability greater than $3 / 4$,

$$
n / 2-c \sqrt{n} \leq|I|=n-\left|\left\{X_{1}, \ldots, X_{k}\right\}\right| \leq n / 2+c \sqrt{n}
$$

for some absolute constant $c>0$. Choosing $\delta=1 / 2$, we get that with a positive probability, both inequalities (3.4) and (3.7) are satisfied. This concludes the proof of the second point of the Proposition 1.

Proof of Theorem 3. For any $p \in(1,2)$, Hölder inequality states that for $\theta=(2-p) / p$

$$
\|f\|_{L_{p}} \leq\|f\|_{L_{1}}^{\theta}\|f\|_{L_{2}}^{1-\theta}
$$

Let $\mu=L \sqrt{n / k} \sqrt{\log k}$ and choose $p=1+1 / \log \mu$. Using Proposition 1, there is a subset $I$ of cardinality greater than $n-k$ for which

$$
\left\|\sum_{i \in I} a_{i} \varphi_{i}\right\|_{L_{2}} \leq C_{p} \mu\left\|\sum_{i \in I} a_{i} \varphi_{i}\right\|_{L_{p}}
$$

where $C_{p}=C /(p-1)^{5 / 2}$. By the choice of $p$ and Hölder inequality,

$$
\left\|\sum_{i \in I} a_{i} \varphi_{i}\right\|_{L_{2}} \leq \mu C_{p}^{p /(2-p)} \mu^{2(p-1) /(2-p)}\left\|\sum_{i \in I} a_{i} \varphi_{i}\right\|_{L_{1}} \leq C \mu h(\mu)\left\|\sum_{i \in I} a_{i} \varphi_{i}\right\|_{L_{1}}
$$

where $h(\mu)=(\log \mu)^{5 / 2}$ and $C$ is an absolute constant.

The same argument works for the second part of the Theorem 3. 


\section{Appendix}

As we mentioned in the Introduction, from a result of J. Bourgain Theorem 3 is "almost" optimal. We would like to thank L. Rodriguez-Piazza for showing us the details of the proof of this optimality in the case of the Walsh system. We consider the Walsh system on $L_{2}[0,1]$ and take the $n$ first functions with $n=2^{N}$. In that case, it is well known that $\left(\varphi_{1}, \ldots, \varphi_{n}\right)$ form a commutative multiplicative group that we denote by $(G, \cdot)$. The main result is based on constructing in any subset of a group a translate of a subgroup of big cardinality.

Lemma 4. Let $(G, \cdot)$ be the multiplicative group generated by the $2^{N}$ first Walsh functions. For any $c \in(0,1)$ and for any subset $\Lambda \subset G$ with $|\Lambda| \geq c 2^{N}$, we can find $b \in G$ and a subgroup $\Gamma$ of $G$ such that, whenever $\log (1 / c) \geq 1 / 2^{N / 2}$,

$$
\begin{cases}\text { 1. } & |\Gamma|=2^{p} \geq N \log 2 /(3 \log (1 / c)) \\ \text { 2. } & b \cdot \Gamma \subset \Lambda .\end{cases}
$$

Assuming this result, we are able to prove the almost optimality of Theorem 3 in the case of the Walsh system $\left(\varphi_{1}, \ldots, \varphi_{n}\right)$ with $n=2^{N}$. Let $I$ be a subset of cardinality $n-k$ with $k \geq \sqrt{n}$. Taking $c=1-k / n$, we have $\log (1 / c) \geq k / n \geq 1 / \sqrt{n}$ and Lemma 4 states that there exists $b \in\left(\phi_{i}\right)_{i=1}^{n}$ and a subgroup $\Gamma$ of $G$ such that $b \cdot \Gamma \subset\left(\phi_{i}\right)_{i \in I}$ and $|\Gamma| \geq(n \log n) /(20 k)$. However, on any subgroup $\Gamma$ of $G$, the $L_{1}$ norm and the $L_{2}$ norm can not be compared with a better estimate than $\sqrt{|\Gamma|}$ since

$$
\left\|\sum_{\gamma \in \Gamma} \gamma\right\|_{L_{2}}=\sqrt{|\Gamma|}\left\|\sum_{\gamma \in \Gamma} \gamma\right\|_{L_{1}} .
$$

Therefore, inequality (3.1) can not be satisfied with a better constant than $\sqrt{n \log n / 20 k}$. In particular when $k$ is proportional to $n$, the factor $\sqrt{\log n}$ is necessary in (3.1).

Proof of Lemma 4. We will prove the result in the case of the abelian additive group $G=\left(\{0,1\}^{N},+\right)$. Note that this lands to a minor change of notation. Let $n=2^{N}$ be the cardinality of this group.

Let $\gamma_{1} \in G \backslash\{0\}$ be such that $\left|\left(\gamma_{1}+\Lambda\right) \cap \Lambda\right|$ is maximal and define $\Lambda_{1}=\left(\gamma_{1}+\Lambda\right) \cap \Gamma$. Applying convolution, it is evident that

$$
\sum_{g \in G}|(g+\Lambda) \cap \Lambda|=|\Lambda|^{2}
$$


and thus

$$
\begin{aligned}
\left|\Lambda_{1}\right| \geq \frac{1}{n-1} \sum_{g \in G \backslash\{0\}}|(g+\Lambda) \cap \Lambda|=\frac{|\Lambda|^{2}-|\Lambda|}{n-1} & \geq c^{2} n \frac{1-1 / c n}{1-1 / n} \\
& \geq c^{2} n\left(1-\frac{1}{c n}\right) .
\end{aligned}
$$

For notational convenience, we define $\gamma_{0}=0$ and $\Lambda_{0}=\Lambda$. We iterate this procedure to construct a family of points $\gamma_{j}$ and sets $\Lambda_{j}$ such that

$$
\left\{\begin{array}{l}
\text { (i) } \quad \gamma_{j} \notin \operatorname{gr}\left\{\gamma_{1}, \ldots, \gamma_{j-1}\right\} \\
\text { (ii) } \quad \Lambda_{j}=\left(\gamma_{j}+\Lambda_{j-1}\right) \cap \Lambda_{j-1} \text { has maximal cardinality } \\
\text { (iii) } \quad\left|\Lambda_{j}\right| \geq c^{2^{j}} n\left(1-\frac{2^{j-1}}{n}\left(1 / c+\cdots+1 / c^{2^{(j-1)}}\right)\right)
\end{array}\right.
$$

where $\operatorname{gr}\left\{\gamma_{1}, \ldots, \gamma_{j-1}\right\}$ is the group generated by $\left\{\gamma_{1}, \ldots, \gamma_{j-1}\right\}$. Once $\gamma_{j-1}$ and $\Lambda_{j-1}$ are constructed, we again use the fact that

$$
\sum_{g \in G}\left|\left(g+\Lambda_{j-1}\right) \cap \Lambda_{j-1}\right|=\left|\Lambda_{j-1}\right|^{2}
$$

and deduce that

$$
\sum_{g \in G \backslash \operatorname{gr}\left\{\gamma_{1}, \ldots, \gamma_{j-1}\right\}}\left|\left(g+\Lambda_{j-1}\right) \cap \Lambda_{j-1}\right| \geq\left|\Lambda_{j-1}\right|^{2}-2^{j-1}\left|\Lambda_{j-1}\right| .
$$

Therefore, there exists $\gamma_{j} \notin \operatorname{gr}\left\{\gamma_{1}, \ldots, \gamma_{j-1}\right\}$ and $\Lambda_{j}=\left(\gamma_{j}+\Lambda_{j-1}\right) \cap \Lambda_{j-1}$ of maximal cardinality such that

$$
\left|\Lambda_{j}\right| \geq\left|\Lambda_{j-1}\right| \frac{\left|\Lambda_{j-1}\right|-2^{j-1}}{n-2^{j-1}}
$$

and (iii) follows from a straightforward computation.

This construction can be continued as long as

$$
1-\frac{2^{p-1}}{n}\left(1 / c+\ldots+1 / c^{2^{(p-1)}}\right)>0
$$

This means that if $p$ is an integer such that

$$
n>p 2^{(p-1)} / c^{2^{(p-1)}}
$$

then $\Lambda_{p} \neq \emptyset$. Let $b \in \Lambda_{p}$, we shall prove that

$$
b+\operatorname{gr}\left\{\gamma_{1}, \ldots, \gamma_{p}\right\} \subset \Lambda .
$$


Indeed let $x \in b+\operatorname{gr}\left\{\gamma_{1}, \ldots, \gamma_{p}\right\}$ and define $\left(\varepsilon_{1}, \ldots, \varepsilon_{p}\right) \in\{0,1\}^{p}$ such that $x=b+\sum \varepsilon_{i} \gamma_{i}$. Since $b \in \Lambda_{p}=\Lambda_{p-1} \cap\left(\gamma_{p}+\Lambda_{p-1}\right)$, there exists $\lambda_{p-1} \in \Lambda_{p-1}$ such that $b=\varepsilon_{p} \gamma_{p}+\lambda_{p-1}$. If one repeats the same argument for $\lambda_{p-1}$ instead of $b$, then at the last step, we get $\lambda_{1} \in \Lambda_{1}=\Lambda \cap\left(\gamma_{1}+\Lambda\right)$ and again, there exists $\lambda \in \Lambda$ such that $\lambda_{1}=\varepsilon_{1} \gamma_{1}+\lambda$. Summarizing, we have found $\lambda \in \Lambda$ such that

$$
b=\lambda+\sum_{i=1}^{p} \varepsilon_{i} \gamma_{i}
$$

Therefore

$$
x=b+\sum_{i=1}^{p} \varepsilon_{i} \gamma_{i}=\lambda \in \Lambda .
$$

To conclude, we have to notice that if $p$ is chosen to be the integer such that $2^{p} \geq \frac{N \log 2}{3 \log (1 / c)}>2^{p-1}$ then

$$
p 2^{(p-1)}\left(\frac{1}{c}\right)^{2^{(p-1)}}<\frac{N^{2} \log 2}{3 \log (1 / c)}\left(\frac{1}{c}\right)^{N \log 2 / 3 \log (1 / c)} \leq N^{2} 2^{N / 2+N / 3} \leq 2^{N}
$$

using the fact that $\log (1 / c) \geq 1 / 2^{N / 2}$. The inequality (3.8) is satisfied and Lemma 4 holds true.

Remark. We would like to thank the referee for pointing out to us that the bound of Theorem 3 is almost optimal for the trigonometric system as well. Indeed, let $n=2^{N}$ and let $\phi_{j}(t)=\exp (2 i \pi j t)$ for $j=0, \ldots, n-1$. Define a one-to-one mapping $F:\{0,1\}^{N} \rightarrow\{0, \ldots, n-1\}$ by

$$
F\left(\varepsilon_{1}, \ldots, \varepsilon_{N}\right)=\sum_{j=1}^{N} \varepsilon_{j} 2^{j-1}
$$

Let $I \subset\{0, \ldots, n-1\}$ be a subset of cardinality $n-k$. By Lemma 4 , the set $\Lambda=F^{-1}(I)$ contains $b \cdot \Gamma$ where $\Gamma=\operatorname{gr}\left(\gamma_{1}, \ldots, \gamma_{p}\right)$. This means that for any set $J \subset\{1, \ldots, p\}, F(b)+\sum_{j \in J} F\left(\gamma_{j}\right) \in I$. Therefore, the function

$$
\psi(t)=\phi_{F(b)} \prod_{j=1}^{p}\left(1+\phi_{F\left(\gamma_{j}\right)}\right)
$$

is contained in $\operatorname{span}\left(\phi_{j}, j \in J\right)$. Obviously,

$$
\|\psi\|_{L_{2}}=\sqrt{|\Gamma|}\|\psi\|_{L_{1}} .
$$




\section{References}

[1] Bourgain, J., Pajor, A., Szarek, S. J. And Tomczak-JaegerMANN, N.: On the duality problem for entropy numbers of operators. In Geometric Aspects of Functional Analysis (1987-88), 50-63. Lecture Notes in Math. 1376. Springer, Berlin, 1989.

[2] Candes, E. And TaO, T.: Near-optimal signal recovery from random projections: universal encoding strategies? IEEE Trans. Inf. Theory $\mathbf{5 2}$ (2006), no. $12,5406-5425$.

[3] CARL, B.: Inequalities of Bernstein-Jackson-type and the degree of compactness of operators in Banach spaces. Ann. Inst. Fourier (Grenoble) 35 (1985), no. 3, 79-118.

[4] Clarkson, J. A.: Uniformly convex spaces. Trans. Amer. Math. Soc. 40 (1936), no. 3, 396-414.

[5] Giné, E. And Zinn, J.: Some limit theorems for empirical processes. Ann. Probab. 12 (1984), no. 4, 929-998.

[6] Guédon, O., Mendelson, S., Pajor, A. and Tomczak-JaegerMANN, N.: Subspaces and orthogonal decompositions generated by bounded orthogonal systems. Positivity 11 (2007), no. 2, 269-283.

[7] Guédon, O. and Rudelson, M.: $L_{p}$-moments of random vectors via majorizing measures. Adv. Math. 208 (2007), no. 2, 798-823.

[8] Lindenstrauss, J. And Tzafriri, L.: Classical Banach spaces. II. Function spaces. Ergebnisse der Mathematik und ihrer Grenzgebiete [Results in Mathematics and Related Areas] 97. Springer-Verlag, Berlin-New York, 1979.

[9] Maurey, B.: Type, cotype and $K$-convexity. In Handbook of the geometry of Banach spaces, Vol. 2, 1299-1332. North-Holland, Amsterdam, 2003.

[10] Maurey, B. And Pisier, G.: Séries de variables aléatoires vectorielles indépendantes et propriétés géométriques des espaces de Banach. Studia Math. 58 (1976), no. 1, 45-90.

[11] Pisier, G.: Martingales with values in uniformly convex spaces. Israel $J$. Math. 20 (1975), no. 3-4, 326-350.

[12] Pisier, G.: The volume of convex bodies and Banach space geometry. Cambridge Tracts in Mathematics 94. Cambridge University Press, Cambridge, 1989.

[13] Rodriguez-Piazza, L.: Personal communication.

[14] Rudelson, M.: Random vectors in the isotropic position. MSRI preprint (1996).

[15] Rudelson, M.: Random vectors in the isotropic position. J. Funct. Anal. 164 (1999), no. 1, 60-72.

[16] Rudelson, M. And Vershynin, R.: On sparse reconstruction from Fourier and Gaussian measurements. Comm. Pure Appl. Math. 61 (2008), no. $8,1025-1045$. 
[17] Sudakov, V. N.: Gaussian random processes and measures of solide angles in Hilbert spaces. Soviet. Math. Dokl. 12 (1971), 412-415.

[18] Talagrand, M.: Sections of smooth convex bodies via majorizing measures. Acta Math. 175 (1995), no. 2, 273-300.

[19] Talagrand, M.: Majorizing measures: the generic chaining. Ann. Probab. 24 (1996), no. 3, 1049-1103.

[20] Talagrand, M.: Selecting a proportion of characters. Israel J. Math. 108 (1998), 173-191.

Recibido: 7 de noviembre de 2007

Olivier Guédon

Université Pierre et Marie Curie, Paris 6 Institut de Mathématiques de Jussieu 4 place Jussieu, 75005 Paris, France guedon@math.jussieu.fr

Shahar Mendelson CMA, The Australian National University Canberra, ACT 0200, Australia and

Department of Mathematics, Technion Haifa, 32000, Israel shahar.mendelson@anu.edu.au

Alain Pajor Université Paris-Est Équipe d'Analyse et Mathématiques Appliquées 5, boulevard Descartes, Champs sur Marne 77454 Marne-la-Vallée, Cedex 2, France Alain.Pajor@univ-mlv.fr

Nicole Tomczak-Jaegermann Dept. of Math. and Stat. Sciences University of Alberta Edmonton, Alberta, Canada, T6G 2G1 nicole.tomczak@ualberta.ca

The second author is partially supported by an Australian Research Council Discovery grant and an Israel Sciences foundation grant 666/06. The fourth author holds the Canada Research Chair in Geometric Analysis. 